\title{
Development of a Revised Performance-Perceptual Test Using Quick Speech in Noise Test Material and Its Norms
}

DOI: $10.3766 /$ jaaa.18059

Hua $\mathrm{Ou}^{*}$

Matthew Wetmore $\dagger$

\begin{abstract}
Background: Two audiometric speech measures have been recognized to have associations with hearing aid use success: the Quick Speech in Noise (QuickSIN) test and the Performance-Perceptual Test (PPT). The PPT involves using the same speech test material (Hearing in Noise Test [HINT]) twice, to evaluate patients' objective and subjective speech recognition performance in noise and the discrepancy between the two measures (Performance-Perceptual Discrepancy [PPDIS]). Using the QuickSIN to conduct the PPT (revised-PPT) may provide clinicians two pieces of important information from one test to help predict hearing aid use success and the need for counseling. Moreover, we could achieve the desired clinical efficiency without purchasing additional test materials.
\end{abstract}

Purpose: This study aimed (1) to evaluate the validity and reliability of using the QuickSIN speech material to administer the PPT and (2) to establish normative data across listeners with normal hearing $(\mathrm{NH})$ and hearing loss $(\mathrm{HL})$.

Research Design: This study used a repeated measures design.

Study Sample: Of the total 65 participants between 18 and 88 years of age, 20 (31\%) had NH and 45 $(69 \%)$ had sensorineural HL, ranging from mild to profound in both ears. Thirty-two of the 45 participants with $\mathrm{HL}$ were hearing aid users.

Data Collection and Analysis: All participants completed the original PPT using HINT and the revisedPPT using QuickSIN, via sound field. Generalized linear mixed models were used to compare the performance, perceptual, and PPDIS data between the two test materials across all participants. Normative data for the revised-PPT were established from all participants.

Results: Significant main effects for both the test material and hearing status were found for the performance and perceptual data. All interactions were nonsignificant. There were no significant PPDIS differences between the original PPT and the revised-PPT. Normative values for the revised-PPT were established and comparable to the norms for the original PPT in the present study. The test-retest results suggested that the revised-PPT has good reliability. In addition, it appeared that there was a negative association between underestimation of hearing ability and hearing aid use success.

Conclusions: It is concluded that the QuickSIN speech material can replace HINT to measure PPT. The revised-PPT may serve as a useful and efficient clinical tool in any clinics for hearing aid fitting and counseling.

Key Words: hearing aid, hearing loss, Performance-Perceptual Test, Quick Speech in Noise test, speech recognition performance

Abbreviations: IOI-HA = International Outcome Inventory for Hearing Aids; 4F-PTA = four-frequency pure-tone average; HINT = Hearing in Noise Test; HL = hearing loss; ICC = intraclass correlation coefficient; $\mathrm{NH}=$ normal hearing; PPDIS = Performance-Perceptual Discrepancy; PPT = PerformancePerceptual Test; QuickSIN = Quick Speech in Noise test; SNR = signal-to-noise ratio; SD = standard deviation

*Department of Communication Sciences and Disorders, Wayne State University, Detroit, MI; †Happy Ears Hearing Center, Phoenix, AZ Corresponding author: Hua Ou, Department of Communication Sciences and Disorders, Wayne State University, Detroit, MI 48202; Email: huou@wayne.edu

This research study was supported in part by the American Speech-Language-Hearing Association's Advancing Academic-Research Careers Award to Dr. Hua Ou.

Partial data were previously presented at the International Hearing Aid Research Conference (IHCON) in Tahoe City, CA, August 10-14, 2016. 


\section{INTRODUCTION}

$\mathrm{H}$ earing loss (HL) is one of the most common conditions in adults. It has been shown that HL can impair a person's participation in daily social activities and may lower his or her quality of life. Hearing aids remain an effective option for people with HL and are associated with improvement of social and/or psychological functioning (e.g., Chisolm et al, 2007). However, approximately one-fourth or less of hearing-impaired people are using hearing aids (e.g., Fischer et al, 2011; Chien and Lin, 2012). Using a prehearing aid-fitting test battery to predict patient success with hearing aids could help clinicians make decisions about appropriate hearing aid technology and guide counseling for patients. Furthermore, clinical efficiency is important to clinicians when considering a test battery. If we could modify a test that is already commonly used in the clinic to collect more information, we may be able to achieve the desired clinical efficiency without purchasing additional test materials.

There are several components throughout the overall hearing aid-fitting process. The primary areas include the selection of hearing aids and features, verification of the fitting, and validation of real-world performance. Prehearing aid fitting in the clinical setting typically includes pure-tone audiometry, immittance measures, and word recognition testing; however, Mueller et al (2010) suggested additional prehearing aid-fitting measures should be included, such as loudness discomfort, speech understanding in quiet and/or in noise, noise annoyance, central auditory processing, cognitive function, patient expectations, and personality assessment. One of the main reasons is that those measures at the prehearing aid-fitting stage may help predict hearing aid use success. For example, Walden and Walden (2004) found unaided Quick Speech in Noise (QuickSIN; Killion et al, 2004) test scores to be a useful tool in predicting hearing aid use success. Another test, the Performance-Perceptual Test (PPT; Saunders et al, 2004), has been shown to provide information about a listener's ability to accurately estimate how well they can understand speech in noise, which is found to be associated with the listener's satisfaction with hearing aids (Saunders et al, 2004; Saunders, 2009).

PPT is a measure in which objective and subjective evaluations are made by using the same test materials, the same test format, and the same unit of measure (signal-to-noise ratio [SNR]). The speech material and competing noise from the Hearing in Noise Test (HINT; Nilsson et al, 1994) using an adaptive procedure are applied to measure the performance and the perceptual SNR-50s. The performance SNR-50 is defined as the
SNR at which the listener repeats the material correctly $50 \%$ of the time. The perceptual SNR-50 is measured based on the perception of the listener to indicate whether he or she understands the sentence. A third result, the Performance-Perceptual Discrepancy (PPDIS), is used as a measure of the listener's ability to accurately assess his or her hearing ability. The PPDIS can be calculated by subtracting the perceptual SNR-50 from the performance SNR-50. If the listener's perceptual SNR-50 is lower (better) than the performance, then he/she overestimates his/her hearing ability. If the perceptual SNR-50 is higher (poorer) than the performance, then he/she underestimates his/her hearing ability. The PPT results help detect participants who significantly under-/overestimate their ability to understand speech in noise, based on normative values (Saunders et al, 2004; Saunders and Forsline, 2006). The information from under-/overestimating listening ability in noise is needed to implement counseling during or after the hearing aid-fitting process (Saunders and Forsline, 2012). Patients who underestimated their ability to listen in noise were more likely to report hearing aid dissatisfaction (Saunders et al, 2004; Saunders, 2009). Saunders et al (2009) and Saunders and Forsline (2012) found that by using counseling to address those who under-/overestimated, patients reported improved perceived hearing ability and improved satisfaction without any changes to hearing aid programming. Because the PPT allows for the detection of individuals who significantly under-/overestimate their ability to understand speech in noise, it provides opportunities for clinicians to implement counseling on realistic expectations for those patients (Saunders, 2009; Saunders and Forsline, 2012). Taken together, the PPT has substantial potentials to be used in clinic.

However, the utility of the PPT using HINT in predicting perceived hearing aid benefit may be limited by the types of speech materials and background noise used. Mueller (2010) conducted a questionnaire with 107 hearing aid dispensers and audiologists, $80 \%$ being audiologists, and found that the QuickSIN was used the most (33\% of the respondents), with $43 \%$ stating that they would probably start using it. More important, the QuickSIN has been found to be a good predictor of hearing aid use success (e.g., Walden and Walden, 2004). The competing signal (four-talker babble) used in the QuickSIN is more representative of real-world listening environments, compared with the speechshaped noise used in the HINT (Killion and Villchur, 1993; Sperry et al, 1997). Using the QuickSIN speech material to conduct the PPT, as a modified test, may allow for the collection of two pieces of information from one test that is already commonly used in the clinic. This combination of information can provide clinicians with more information from administering one test rather than two. 
The aim of this study was to:

- evaluate the validity and reliability of using the QuickSIN speech material to administer the PPT and

- establish normative data across listeners with normal hearing $(\mathrm{NH})$ and $\mathrm{HL}$.

\section{METHOD}

\section{Participants}

Fifty-four participants with HL were recruited, and 45 were eligible with the following inclusion criteria: (a) adults older than 18 years; (b) bilateral sensorineural HL (air-bone gap $<10 \mathrm{~dB}$ ); (c) hearing symmetry, that is, an interaural threshold difference in the hearing level of $<15 \mathrm{~dB}$ across the frequencies $500,1000,2000$, and $4000 \mathrm{~Hz}$; (d) hearing threshold levels no better than 20-dB HL at $500 \mathrm{~Hz}$ and no worse than 75-dB HL at $3000 \mathrm{~Hz}$ (re: ANSI, 1996); and (e) normal tympanograms. In addition, 20 participants with $\mathrm{NH}$ (both air and bone conduction thresholds $<25-\mathrm{dB}$ HL across octave frequencies of 250-8000 $\mathrm{Hz}$ ) were recruited for the study. A total of 65 participants between the ages of 18 and 88 years (mean = 45.0 years; standard deviation $[\mathrm{SD}]=22.0$ years) participated in the study. Thirty-two of the 45 participants with HL were hearing aid users. Among hearing aid users, 30 were fitted bilaterally. The average length of hearing aid use experiences was 9.7 years (range $=6$ weeks -40 years). The different styles included behind-the ear $(\mathrm{n}=6)$, receiver-in-canal $(\mathrm{n}=$ $21)$, in-the-canal $(n=1)$, and in-the-ear $(n=4)$ hearing aids. Figure 1 shows the mean air conduction thresholds, for all participants, from 250 to 8000
Hz. Details of the demographic and audiological information are displayed in Table 1.

Participants were recruited through fliers posted in local clinics and communities. $\mathrm{NH}$ participants were recruited on a volunteer basis and were not compensated for their time. Participants who met the criteria for HL and hearing aid experience were compensated for their participation. The Institutional Review Board from Illinois State University (both authors' previous affiliation) approved this project.

\section{Equipment Setup}

Testing took place in a sound-treated booth. The speech stimuli and noise were played from a computerbased 2-channel audiometer (MADSEN Astera; GN Otometrics A/S, Taastrup, Denmark) and an amplifier (ART SLA-2; Sweetwater Sound, Fort Wayne, IN), and then presented through the loudspeaker (Reveal 601P; Tannoy Limited, Coatbridge, Scotland) from $0^{\circ}$ azimuth and at $0^{\circ}$ elevation. The distance between the loudspeaker and the participant was $1 \mathrm{~m}$.

\section{Test Measures}

\section{Audiometric Procedures}

After the participant consented to participate in the study, otoscopy and tympanometry were conducted. Hearing status was measured by pure-tone audiometry following the American Speech-Language-Hearing Association-recommended procedure in a sound-treated booth. Pure-tone air (octave frequencies of $250-8000 \mathrm{~Hz}$ ) and bone conduction (octave frequencies of $500-4000 \mathrm{~Hz}$ ) audiometric testing was performed, bilaterally, for all participants. A four-frequency pure-tone average (4FPTA) was calculated for each ear using 500, 1000, 2000,

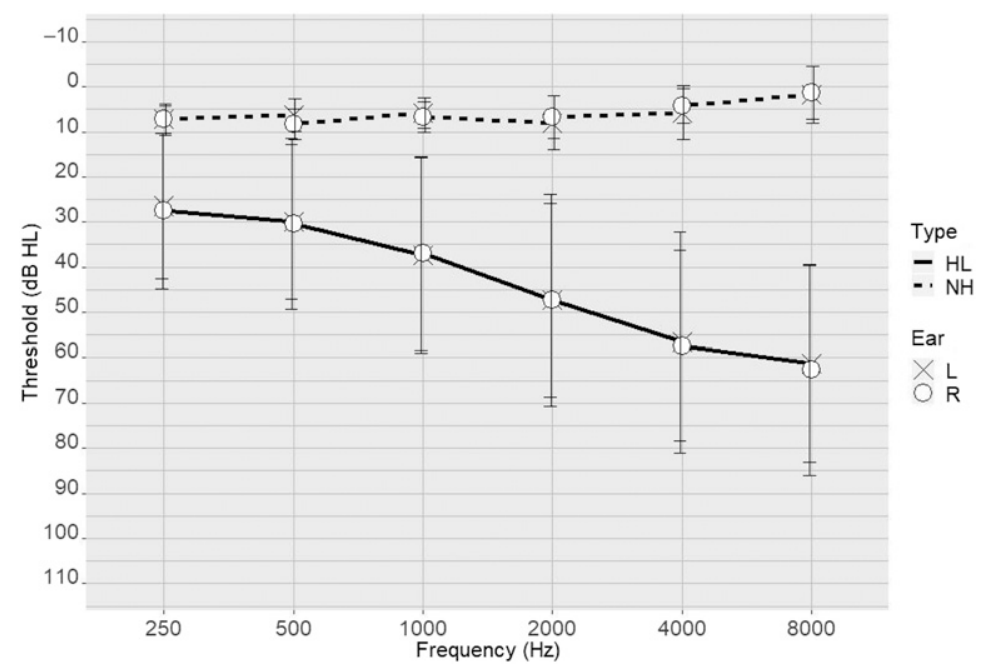

Figure 1. Mean pure-tone thresholds for the left $(\mathrm{X})$ and right $(\mathrm{O})$ ears in $\mathrm{dB}$ HL for groups with NH and hearing impairment. The error bars show one standard deviation. 
Table 1. Demographic Information of the Participants

\begin{tabular}{|c|c|c|c|c|c|}
\hline \multirow[b]{2}{*}{ Participants } & & \multicolumn{2}{|c|}{ Gender } & \multicolumn{2}{|c|}{ Age (years) } \\
\hline & & Male & Female & Mean & SD \\
\hline \multicolumn{2}{|l|}{$\overline{\mathrm{NH}}(\mathrm{n}=20)$} & 2 & 18 & 23.3 & 6.7 \\
\hline \multirow[t]{2}{*}{$\mathrm{HL}(\mathrm{n}=45)$} & $\mathrm{HA}(\mathrm{n}=32)$ & 14 & 18 & 54.0 & 19.8 \\
\hline & Non-HA $(n=13)$ & 7 & 6 & 63.8 & 10.5 \\
\hline
\end{tabular}

and $4000 \mathrm{~Hz}$. Participants were categorized as NH (4F$\mathrm{PTA}=<25 \mathrm{~dB} \mathrm{HL}$ ) and HL (4F-PTA $>25 \mathrm{~dB} \mathrm{HL}$ ).

\section{PPT Materials}

Original PPT with HINT: The original PPT used the HINT speech material, adaptive protocol, test materials, and speaker configuration. The ten-sentence lists were used along with a competing steady-state noise shaped to the average long-term spectrum of the sentences in the current study.

\section{Original PPT Instructions to Participants}

Perceptual Condition:

\begin{abstract}
"This is a test of your ability to hear soft speech in a noisy situation. First, you will hear background noise. Then, you will hear a man reading a sentence. The loudness of the man's voice will change during testing. Sometimes it will be very faint. Listen closely to each sentence and let me know whether you understood the ENTIRE sentence-with a yes or no response."

Performance Condition:

"Now, you will listen to the same sentences, only this time you will be asked to repeat what the man says. Repeat everything you hear the man says even when his voice is very soft. I will stop after each sentence to allow you to repeat what you heard. Please repeat everything you hear, even if it is only part of the sentence. It is all right to guess."
\end{abstract}

Revised-PPT with QuickSIN: The revised-PPT used the same PPT procedure, but instead of HINT as the speech material, the QuickSIN speech material was used. The six-sentence lists with five key words per sentence were used along with four-talker babble noise.

\section{Revised-PPT Instructions to Participants}

Perceptual Condition:

\footnotetext{
"Imagine that you are at a party. There will be a woman talking and several other talkers in the background. The woman's voice is easy to hear at first because her voice is louder than the others. The background talkers will gradually become louder, making it difficult to understand the woman's voice. Listen closely to each sentence and let us know how much you are able to understand based on a scale from 0 to 5 . Zero means that you understand nothing from the sentence. Five means that you can understand every word for the
}

whole sentence. Two-and-a-half means that you can only understand half of the sentence. Please use any number between 0 and 5 to indicate how much you understood the sentence. The noise will start first, and then you will hear the woman's voice."

Performance Condition:

"Now, you will listen to the same sentences; only this time you will be asked to repeat what the woman says. Please guess and repeat as much of each sentence as possible. The noise will start first and then you will hear the woman's voice."

\section{Procedures of the PPT}

If the participant was eligible to be enrolled in the study, the test commenced.

Original PPT: We followed the same procedure as in Saunders et al (2004). That is, the HINT protocol (Nilsson et al, 1994) using an adaptive procedure was followed. The presentation level for noise was fixed at $65 \mathrm{dBA}$, whereas the speech level varied depending on the responses. If the entire sentence was repeated back correctly, the speech level was decreased to make the SNR more adverse. The initial sentence was played at $4 \mathrm{~dB}$ below the noise level. The step adjustment started with 4-dB steps for the first four sentences and then 2-dB steps for the rest of the sentences. Two ten-sentence lists of 25 lists were administered for each participant per condition. The result for one condition was based on the average presentation levels of the fifth to twenty-first sentence subtracted by the noise level of $65 \mathrm{dBA}$. The SNR-50 was based on the average results of two lists. In the perceptual condition, the participant was asked to say "yes" or "no" to indicate whether he or she can understand the entire sentence. In the performance condition, the participant was asked to repeat back the entire sentence. The test always started with the perceptual condition followed by the performance condition as suggested in Saunders et al (2004) and Saunders and Forsline (2006). The same lists were used for both conditions. The difference between the performance and perceptual SNR-50s was used to determine the PPDIS, which represents a measure of the participant's ability to accurately assess his or her hearing ability.

Revised-PPT: Both speech and noise were presented from the same channel and the same loudspeaker. The stimuli were presented at predetermined SNRs, which decrease in 5 - $\mathrm{dB}$ steps from 25 (very easy) to 0 (extremely difficult). The presentation level was set at $70-\mathrm{dB}$ HL. If the participant's PTA was $>45-\mathrm{dB}$ $\mathrm{HL}$, the stimuli were presented at a level that the participant perceived to be "loud, but ok." For the current study, only the lists with equivalent difficulty were used (i.e., lists 1, 2, 6, 8, 10,11, 12, 15, and 17 as suggested in 
McArdel and Wilson, 2006). Because the stimuli were prerecorded at predetermined SNRs, the levels for speech and noise were not manually adjusted. Again, the measure of the perceptual SNR-50 was always conducted before that of the performance SNR-50. The participant indicated if he/she can understand the entire sentence by using a six-point scale to the sentence presented. The scale was from 0 to 5 . The number " 0 " indicated that the participant understood nothing from the sentence. The number " 5 " indicated that the participant can understand every word of the whole sentence, which also indicated the person can understand the five key words. The number two-and-a-half indicated that the participant can only understand half of the sentence (i.e., 2.5 key words). The participant could use any number between zero and five to indicate how much he/she understood the sentence. Each number being assigned to each sentence suggested the number of correct key words. The formula " 27.5 - (total key words correct)" was used to calculate the perceptual SNR-50 for each list (please refer to Killion et al, 2004 for where the number 27.5 comes from). A total of four lists with 24 sentences were tested to obtain the average perceptual SNR-50 for each participant. For the measurement of the performance SNR-50, the same procedure was repeated, but instead of using a scale, the participant repeated back the sentence and the number of correct key words was recorded. The same four lists as in the perceptual condition were applied to obtain the performance SNR-50. Moreover, by subtracting the perceptual SNR-50 from the performance SNR-50, the PPDIS was obtained for the revised-PPT.

The order of the sentence lists was counterbalanced across participants with NH and HL for both the original and revised-PPT. The order of the original and revised-PPT was also counterbalanced between participants. Participants were given breaks, as needed. The retest condition for the revised-PPT was conducted at the end of the session for each participant with HL. Four different lists of QuickSIN sentences were applied compared with the initial test. Hearing aid users completed all test conditions unaided.

\section{Hearing Aid Use Success Measured by International Outcome Inventory for Hearing Aids (IOI-HA)}

The seven-item IOI-HA (Cox and Alexander, 2002) was administered using paper and pencil for hearing aid users. It is of interest to evaluate the relationship between hearing aid use success and the listener's ability to judge how accurately he/she can hear based on the results from the revised-PPT. Because there is no universal accepted definition of hearing aid use success, we adapted the one from Hickson et al (2014) and made a slightly stricter definition. Hearing aid use success was defined as that the participant reported more than eight hours of hearing aid use daily (Item \#1) and experienced a significant benefit at the situation where he/she wanted to hear better (Item \#2). In other words, the combination of the response " 5 " on Item \# 1 and " 4 or 5 " on Item \#2 of the IOI-HA was considered as hearing aid use success.

\section{Data Analysis}

Group mean perceptual, performance SNR-50, and PPDIS along with SDs were reported. Generalized linear mixed models were used to compare the performance, perceptual, and PPDIS data between the two test materials across participants with $\mathrm{NH}$ and HL. The independent variables were test material (two levels $=$ QuickSIN and HINT), hearing status (two levels $=\mathrm{NH}$ and HL), and interaction between test material and hearing status. The dependent variables were performance, perceptual, and PPDIS for each model. Normative values for the revised-PPT were established using the rule of three following how the norms were developed for the original PPT (as summarized in Mueller et al, 2014). That is, if the PPDIS <33rd percentile of normative data, the person can be defined as an underestimator; if the PPDIS $>66$ th percentile of normative data, the person can be defined as an overestimator; for those between the 33rd and 66th percentile of normative data, the person can be defined as an accurate estimator. The agreement of under-/overestimators was explored between the original and the revised-PPT. Test-retest reliability was assessed using the Pearson correlation and intraclass correlation coefficient (ICC) for the revised-PPT based on the data from participants with HL. For all tests, statistical significance was defined as a $p<0.05$. Data were analyzed using Statistical Analysis System (SAS Institute, Cary, NC) v. 9.4.

\section{RESULTS}

\section{Performance and Perceptual SNR-50}

Table 2 displays detailed descriptive statistics for both the original and the revised-PPT across participants. The average time to conduct the revised-PPT or the original PPT was about eight minutes in the current study.

A series of generalized linear mixed models revealed significant main effects for both the test material $\left[F_{(1,126)}=45.47, p<0.0001\right]$ and hearing status $\left[F_{(1,126)}=\right.$ 25.09, $p<0.0001$ ] when using performance SNR-50 as the dependent variable. No interaction was found between the test material and the hearing status ( $p=$ 0.99). The follow-up analyses with the Tukey-Kramer correction to adjust the $p$-values were applied to answer specific questions within the significant main effects. The performance SNR-50s with HINT were better (lower) than those with QuickSIN $\left[t_{(126)}=-6.74\right.$, 
Table 2. The Average Performance and Perceptual SNR-50 along with the PPDIS for the Original PPT and the Revised-PPT

\begin{tabular}{llrrr}
\hline & \multicolumn{1}{c}{ dB } & Mean & SD & SE \\
\hline Original PPT (HINT) & Performance SNR-50 & $0.80(-2.73)$ & $5.15(1.48)$ & $0.77(0.33)$ \\
& Perceptual SNR-50 & $1.40(-2.93)$ & $5.28(2.80)$ & $0.79(0.63)$ \\
Revised-PPT (QuickSIN) & PPDIS & $-0.60(0.20)$ & $2.48(3.54)$ & $0.37(0.79)$ \\
& Performance SNR-50 & $5.54(2.03)$ & $3.28(1.53)$ & $0.49(0.34)$ \\
& Perceptual SNR-50 & $5.69(1.50)$ & $2.93(1.52)$ & $0.44(0.34)$ \\
& PPDIS & $-0.14(0.53)$ & $2.49(1.70)$ & $0.37(0.38)$ \\
\hline
\end{tabular}

Note: The values in the parentheses were for the participants with $\mathrm{NH}$.

adjusted $p<0.0001]$. Participants with $\mathrm{NH}$ performed better than those with HL $\left[t_{(126)}=-5.01\right.$, adjusted $p<$ 0.0001]. Significant main effects were also found for the test material $\left[F_{(1,126)}=36.99, p<0.0001\right]$ and hearing status $\left[F_{(1,126)}=35.31, p<0.0001\right]$ when using perceptual SNR-50 as the dependent variable. Again, there was no interaction between the test material and hearing status $(p=0.92)$. Follow-up analyses with the Tukey-Kramer adjustment revealed that the perpetual SNR-50s with HINT were better than those with Quick$\operatorname{SIN}\left[t_{(126)}=-6.08\right.$, adjusted $\left.p<0.0001\right]$ as well. Participants with $\mathrm{NH}$ self-perceived better performance than those with HL $\left[t_{(126)}=-5.94\right.$, adjusted $p<$ 0.0001].

\section{PPDIS}

The average PPDIS of the original PPT was -0.60 $(\mathrm{SD}=2.48)$ for participants with HL and -0.14 (SD $=$ 2.49) for the revised-PPT. The average PPDIS reported by Saunders and Forsline (2006) was $-1.4(\mathrm{SD}=3.5)$ for those with HL. Using PPDIS as the dependent variable, the results from the mixed model indicated that there were no significant main effects for the test material $\left[F_{(1,126)}=0.62, p=0.43\right]$ and hearing status $\left.\left[F_{(1,126)}=2.25, p=0.14\right)\right]$. That is, the discrepancies between the performance SNR-50s and the perceptual SNR-50s were not significantly different, regardless of the test material and HL. Saunders et al (2004) also found that the PPDIS was irrelevant to the presentation level and the degree of HL.

Figure 2 displays the histograms of performance SNR-50, perceptual SNR-50, and PPDIS across all participants. It seemed that all three variables were normally distributed around each mean for both test materials. The distribution of PPDIS values for the original and the revised-PPT overlapped with each other.

\section{Normative Data for the Revised-PPT}

The results for the present study placed underestimators with a PPDIS at $\leq-1.0 \mathrm{~dB}$, overestimators at $\geq 0.63 \mathrm{~dB}$, and accurate estimators in between these two values for the revised-PPT when used the rule of three. The same rule was also applied to the original PPT data to develop the norms for the current study. Table 3 displays the detailed normative values for both the original and the revised-PPT.

The absolute agreement was 31 of 65 (47.7\%), which indicated that the ratings were consistent across underestimators, accurate estimators, and overestimators between the two test materials. It should be noted that because HINT and QuickSIN are two different speech tests, we did not expect the absolute agreement to be high when we categorized the participants using the norms. However, because we used the concept of PPT to adapt the QuickSIN speech materials, PPDIS should be the key variable to compare between the original and the revised-PPT in the current study.

For the hearing aid users assessed by the revisedPPT in the present study $(\mathrm{n}=32), 13$ were underestimators, 12 were overestimators, and seven were accurate estimators.

\section{Relationship Between Hearing Aid Use Success and Revised-PPT}

Furthermore, we evaluated the relationship between hearing aid use success and the listener's ability to judge how accurately he/she can hear based on the results from the revised-PPT. Recall that hearing aid use success in the present study was defined as that the participant reported more than eight hours of hearing aid use daily (Item \#1) and experienced a significant benefit at the situation where he/she wanted to hear better (Item \#2). Five of seven (71.4\%) accurate estimators and 9 of $12(75.0 \%)$ overestimators can be categorized as successful hearing aid users, whereas only 5 of 13 $(38.5 \%)$ underestimators were successful hearing aid users.

\section{Test-Retest Reliability for the Revised-PPT}

The test-retest reliability was evaluated based on the participants with HL who were tested twice for the revised-PPT. Pearson correlation revealed that the revised-PPT provided good test-retest reliability 

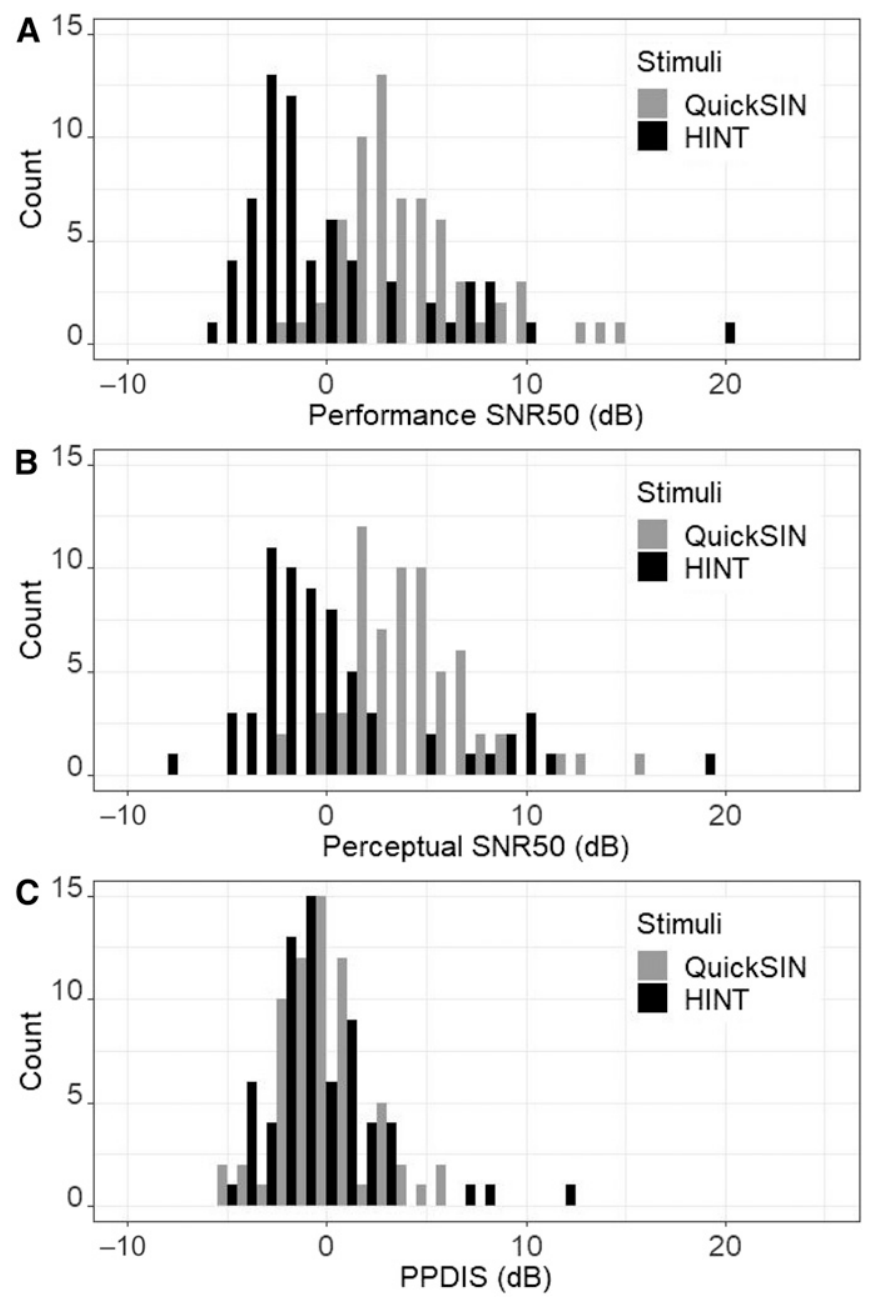

Figure 2. Histograms for performance SNR-50, perceptual SNR-50, and PPDIS between two test materials across the 65 participants.

(performance $r=0.92$; perceptual $r=0.84$; PPDIS $r=$ 0.62 ; all $p$ values $<0.0001$ ). The ICC also revealed good test-retest reliability for the revised-PPT (performance ICC $=0.89$; perceptual $\mathrm{ICC}=0.84$; PPDIS $\mathrm{ICC}=0.53)$.

\section{DISCUSSION}

$\mathrm{T}$ his study investigated the possibility of applying the QuickSIN speech material to conduct the PPT. The results indicated that it is valid and reliable

Table 3. Normative PPT Value Were Established for the Revised-PPT and the Original PPT to Self-Rate Listening in Noise Ability Derived from Participants in this Study

\begin{tabular}{lcc}
\hline Underestimator & Accurate & Overestimator \\
\hline Revised-PPT normative values & $>-1 \&<0.63$ & $\geq 0.63$ \\
$\leq-1$ & $>-1$ s & $\geq 0$ \\
Original PPT normative values from the present study \\
$\leq-1.65$ & $>-1.65 \&<0$ &
\end{tabular}

Note: $\mathrm{n}=65$ to use the QuickSIN speech material for the PPT. The discrepancy between the performance and the perceptual components (PPDIS) of the revised-PPT was not significantly different compared with that of the original PPT in the present study.

The biggest difference between the speech material used in the original and the revised-PPT was the background noise. The revised-PPT used four-talker babble, whereas the original PPT used nonmodulated broadband speech noise, shaped to be similar to the long-term average speech spectrum of the sentences. Because the lack of modulation in steady-state noise has been noted to be less representative of everyday speech in noise situations than babble noise (Wilson et al, 2007), the revised-PPT using QuickSIN has better ecological validity than the original PPT using HINT.

Another difference between the original and the revised-PPT was the procedure to measure the lowest SNR where the individual can understand the entire sentence in noise $50 \%$ of the time, also known as SNR-50. The original PPT using HINT used an adaptive procedure, where the presentation level of the 
competing noise was fixed, and the speech material was varied. The speech presentation level was varied as a function of the individual's performance on the previous sentence and was used to determine the SNR-50. The revised-PPT using QuickSIN also used an adaptive method where the sentences remained at a fixed presentation level and the competing noise was increased at predetermined levels. Although there were substantial differences to measure SNR-50 between the original and the revised-PPT, the concept of PPDIS was identical for both tests. This was supported by the results from the present study.

Regarding the normative data between the original PPT and the revised-PPT, it appeared that the norms for both tests were similar in the current study. Our norms of the original PPT indicated the cutoff PPDIS values of $\leq-1.65$ for underestimators and $\geq 0$ for overestimators. Saunders and her colleagues reported the cutoff values were $\leq-3$ for underestimators and $\geq 0.2$ for overestimators. Although the absolute values were not exactly the same between our study and Saunders et al (2004), the meaning of the PPDIS was the same. That is, underestimators can be defined as those with negative PPDIS values and overestimators can be defined as those with positive PPDIS values (e.g., Saunders and Forsline, 2012). It should be noted that the rule of three to develop the normative data is arbitrary. Therefore, it is necessary to develop norms for different population at different clinics.

The results from the present study indicated that the test-retest reliabilities were slightly lower across three components than those reported in Saunders et al (2004). It is likely due to the reason that a different type of background noise was used in the revised-PPT. The multitalker babble in the revised-PPT has both energetic and informational masking effects, whereas the steady-state noise in the original PPT only causes energetic masking. The energetic masking refers to the physical interference of noise for speech, and the informational masking refers to the perceptual interference of the masking signal on speech. It appeared that the multitalker babble in the revised-PPT might make it more difficult for listeners to judge how much they can understand in noise compared with the steady-state noise in the original PPT.

Regarding the relationship between hearing aid use success and the revised-PPT, it appeared that underestimators were less likely to become successful hearing aid users in the present study. Saunders et al (2004) and Saunders and Forsline (2006) reported that participants with greater self-reported hearing handicap tended to underestimate their hearing ability and more likely to report hearing aid dissatisfaction (e.g., Saunders, 2009). Because there is no universal definition of hearing aid use success, we can only speculate there might be a negative association between underestimation of hearing ability and hearing aid use success. More research is needed in this area.

\section{Clinical Implications}

The current results have several important clinical implications. First, clinicians can use one single test, the revised-PPT, to gather two pieces of important information to aid in determining a patient's potential success with hearing aids. That is, using the QuickSIN speech material to conduct the PPT can allow clinicians to collect the QuickSIN speech recognition performance (SNR loss), as well as the discrepancy between the performance and the perceptual components (PPDIS) from the same test. Second, although speech testing in the booth does not always equate to real-world performance, this will allow clinicians to make the most of clinical efficiency while also obtaining useful information in making decisions regarding hearing aid selections and options. The revised-PPT using QuickSIN can also be useful in the counseling aspect to help patients achieve more benefits without (re)programming hearing aids. Last, the QuickSIN test has been identified as one of the most commonly used speech in noise tests among clinics (Mueller, 2010). It should be effortless to adapt the methodology of the revised-PPT into any clinical setting without obtaining new test materials. In addition, it only required an average of eight minutes to conduct the revised-PPT or the original PPT in the present study. Mueller et al (2014) recommended to add speech recognition in noise tests before hearing aid fitting. If clinicians would like to incorporate one into their clinical practice, the revised-PPT appears to be a good option because of the clinical efficiency.

In summary, it is valid and reliable to measure PPDIS using the QuickSIN speech material compared with the original PPT. The revised-PPT can serve as a useful and efficient clinical tool.

Acknowledgments. We thank all the participants for their time.

\section{REFERENCES}

American National Standards Institute (ANSI). (1996) American National Standard: Specification for Audiometers (ANSI S3.6). New York, NY: ANSI.

Chien W, Lin F. (2012) Prevalence of hearing aid use among older adults in the United States. Arch Intern Med 172:292293.

Chisolm T, Johnson C, Danhauer J, Portz L, Abrams H, Lesner S, McCarthy P, Newman C. (2007) A systematic review of healthrelated quality of life and hearing aids: final report of the American Academy of Audiology Task Force on the Health-Related Quality of Life Benefits of Amplification in Adults. J Am Acad Audiol 18:151-183. 
Cox R, Alexander G. (2002) The International Outcome Inventory for Hearing Aids (IOI-HA): psychometric properties of the English version. Int $J$ Audiol 41:30-35.

Fischer M, Cruickshanks K, Wiley T, Klein B, Klein R, Tweed T. (2011) Determinants of hearing aid acquisition in older adults. Am J Public Health 101:1449-1455.

Hickson L, Meyer C, Lovelock K, Lampert M, Khan A. (2014) Factors associated with success with hearing aids in older adults. Int $J$ Audiol 53(1, Suppl):S18-S27.

Killion M, Niquette P, Gudmundsen G, Revit L, Banerjee S. (2004) Development of a quick speech-in-noise test for measuring signalto-noise ratio loss in normal-hearing and hearing-impaired listeners. J Acoust Soc Am 116:2395-2405.

Killion M, Villchur E. (1993) Kessler was right - partly: but SIN test shows some aids improve hearing in noise. Hear $J$ 46:31-35.

McArdel R, Wilson R. (2006) Homogeneity of the 18 QuickSIN lists. J Am Acad Audiol 17:157-167.

Mueller HG. (2010) Three pre-tests: what they do and why experts say you should use them more. Hear $J$ 63:17-24.

Mueller HG, Johnson EE, Weber J. (2010) Fitting hearing aids: a comparison of three pre-fitting speech tests. Audiology Online https:/www.audiologyonline.com/articles/fitting-hearing-aidscomparison-three-861

Mueller HG, Ricketts TA, Bentler R. (2014) Pre-fitting testing using speech material. In Modern Hearing Aids: Pre-Fitting Testing and Selection Considerations. 1st ed. San Diego, CA: Plural Publishing, 123-193.

Nilsson M, Soli SD, Sullivan JA. (1994) Development of the Hearing in Noise Test for the measurement of speech reception thresholds in quiet and in noise. J Acoust Soc Am 95:1085-1099.

Saunders G. (2009) Understanding in noise: perception vs. performance. Hear $J$ 62:10-16.

Saunders G, Forsline A. (2006) The performance-perceptual test and its relationship to aided reported handicap and hearing aid satisfaction. Ear Hear 27:229-242.

Saunders G, Forsline A. (2012) Hearing-aid counseling: comparison of single-session informational counseling with singlesession performance-perceptual counseling. Int $J$ Audiol 51: 754-764.

Saunders G, Forsline A, Fausti S. (2004) The performance-perceptual test and its relationship to unaided reported handicap. Ear Hear 25: 117-126.

Sperry J, Wiley T, Chial M. (1997) Word recognition performance in various background competitors. $J$ Am Acad Audiol 8:71-80.

Walden T, Walden B. (2004) Predicting success with hearing aids in everyday living. $J$ Am Acad Audiol 15:342-352.

Wilson R, Mcardle R, Smith S. (2007) An evaluation of the BKBSIN, HINT, QuickSIN, and WIN materials on listeners with normal hearing and listeners with hearing loss. J Speech Lang Hear Res 50:844-856. 